\title{
À propos de Les syndicats nationaux... de Jacques Rouillard
}

\section{Stanley Bréhaut Ryerson}

Volume 35, numéro 3, décembre 1981

URI : https://id.erudit.org/iderudit/303977ar

DOI : https://doi.org/10.7202/303977ar

Aller au sommaire du numéro

Éditeur(s)

Institut d'histoire de l'Amérique française

ISSN

0035-2357 (imprimé)

1492-1383 (numérique)

Découvrir la revue

Citer cette note

Ryerson, S. B. (1981). À propos de Les syndicats nationaux... de Jacques

Rouillard. Revue d'histoire de l'Amérique française, 35(3), 397-406.

https://doi.org/10.7202/303977ar d'utilisation que vous pouvez consulter en ligne.

https://apropos.erudit.org/fr/usagers/politique-dutilisation/ 


\section{NOTE CRITIQUE}

\section{À PROPOS DE LES SYNDICATS NATIONAUX... DE JACQUES ROUILLARD*}

Comme en témoigne le Prix Lionel-Groulx décerné à l'auteur, ce volume constitue un apport de première valeur à l'histoire sociographique québécoise et canadienne. Cette reconnaissance bien méritée témoigne également de l'intérêt croissant qu'on porte à l'histoire sociale, et à celle du mouvement ouvrier en particulier (le Prix Macdonald, tout récemment décerné par la Société historique du Canada à Gregory Kealey, auteur de Toronto workers respond to industrialism, en représente, en quelque sorte, la contrepartie au Canada anglais).

L'ouvrage de Jacques Rouillard, à la fois narration bien étoffée, fruit d'une recherche méthodique et exhaustive, et réflection analytique originale, comble une lacune importante de notre historiographie. Car ce qu'on nous propose est à la fois une histoire événementielle et institutionnelle du syndicalisme québécois au premier tiers du siècle; un examen systématique des interrelations complexes des quatre principaux courants qui le composèrent; et un essai d'analyse théorique sur le thème des relations réciproques du nationalisme et du syndicalisme. Le défi est de taille! Si, à mon avis, ce sont les deux premiers éléments qui sont les mieux réussis, alors que le troisième laisse subsister un brin d'interrogation théorique non résolue, j'ai bien hâte d'ajouter que le travail réalisé par l'auteur fait avancer de manière sensible la réflexion indispensable, dans un domaine où, de toute façon, les certitudes sont loin d'être choses acquises.

La structure d'ensemble de l'ouvrage comprend les deux volets, chronologique et analytique, dont les diverses composantes sont imbriquées de façon lucide et intéressante (on ne les sépare ici que pour les fins de recension). Ils se résument ainsi:

- Le «syndicalisme national» à la fin du 19e siècle: Chevaliers du travail, syndicats nationaux non-confessionnels, syndicats

* Rouillard, Jacques, Les syndicats nationaux au Québec de 1900 à 1930. Québec, Les Presses de l'Université Laval, Cahiers d'histoire, no 24, 342 p., 1979. Prix LionelGroulx 1980. 
internationaux (tendance autonomiste au sein du CMTC, réplique de la Fédération américaine du Travail); dix ans de conflits, un tournant: 1892-1902; les syndicats nationaux et les débuts du syndicalisme catholique, 1903-1917; le clergé et le syndicalisme, la CTCC, 1918-1930; les internationaux face au syndicalisme catholique; syndicats catholiques, patronat, gouvernement.

- Idéologies et pratiques syndicales; nationalisme et syndicalisme; catholicisme social, doctrine sociale de l'Église; libéralisme, matérialisme, syndicalisme d'affaires chez les internationaux; mutations et éléments de convergence.

Le propos de l'auteur est de «raconter pourquoi et comment» la force du sentiment national au Québec «s'est conjuguée à celle du syndicalisme pour donner naissance à des syndicats nationaux, d'abord non confessionnels, puis par la suite réservés aux seuls catholiques». Face au problème qui consistait à «concilier la défense de leurs intérêts professionnels avec leur désir de se regrouper sur une base nationale», comment ces syndicats «ont-ils pu marier cette double allégeance syndicale et nationale? Pourquoi, alors que la majorité des travailleurs joignait les rangs de fédérations internationales, une minorité importante a-t-elle préféré se donner des structures nationales?» (Avant-propos, 3).

De sa perception d'ensemble concernant les origines et l'essor initial du syndicalisme ouvrier, Rouillard dégage comme trait spécifique la présence, profondément enracinée au Québec, de la conscience nationale. Qu'il s'agisse des Chevaliers du travail, des syndicats nationaux non-confessionnels ou du syndicalisme catholique, la dimension d'identité ethnique s'affirme - face aux pressions d'une «altérité» externe et perçue comme menaçante. Même au sein du Congrès des métiers et du travail canadien, pourtant affilié à la Fédération américaine du travail, des remous autonomistes se font sentir, se font réprimer, se réaffirment.

Certes, on nous rappelle que ce «national» n'est pas univoque. Il y a eu à la fois le sentiment canadien (pan-canadien), et ce que de nos jours on dénommera québécois (et qui n'est pas sans comporter son ingrédient canadien). On aurait aimé que l'auteur s'attarde un peu plus sur cette particularité de notre situation. Dire simplement que ce nationalisme s'explique par «les différences culturelles» (120) est un peu court. Ces différences recouvrent une dimension cruciale d'inégalité, sans quoi la «question nationale», sa pérennité et ses péripéties s'expliquent difficilement. L'infrastructure des idéologies existe, elle aussi; à la prendre pour acquise, sans questionnement ni curiosité, on risquerait de déboucher sur 
un scénario fort incomplet où ce seraient les idées, à elles seules, qui «mènent le monde».

Rouillard a assurément raison d'insister sur la constante présence, la pression efficace du facteur national: il y retrouve l'explication à la fois des limites auxquelles se sont butés les internationaux au Québec, et de l'ouverture dont a su profiter l'Église, en utilisant le sentiment national à des fins conservatrices, pour édifier un mouvement syndical bien à elle. Sa démonstration de cette thèse d'interprétation est l'un des deux ingrédients les plus importants de son livre; l'autre, à mon sens, est le passage-charnière de son récit qui s'intitule: «Mutation de la CTCC». Déjà, la première décennie d'existence des Syndicats catholiques offre le spectacle anticipé de changements à venir dans le quart de siècle qui suit, lors des grèves de Sorel et puis d'Asbestos: «Bien avant 1950, la CTCC évoluait dans le sens d'une certaine radicalisation...» (249). L'auteur évoque entre autres deux grèves, de 1924 et de 1926. "Ä la compagnie E.B. Eddy de Hull, 275 ouvrières employées à la fabrication d'allumettes et faisant partie d'un syndicat affilié à la CTCC déclenchent une grève parce que l'employeur demande à chacune d'elles un engagement écrit à renoncer au syndicat. Après un mois de grève, la compagnie céda sur tous les points...» (243). Dans la vieille Capitale, deux ans plus tard, une décision patronale de réduire de trente pour cent les salaires des travailleurs de la chaussure provoqua... «l'arrêt de travail de 5000 travailleurs et dura quatre mois, du moins pour certains d'entre eux». Cette grève, «sans contredit le plus important conflit ouvrier à survenir au Québec pendant les années 1920», attira de la part de Mgr Langlois un blâme public au groupe patronal, dont l'Association "avait toujours voulu la destruction des syndicats dans le passé», et un éloge de la «solidarité ouvrière bien comprise et librement appliquée» (243-246).

La «mutation» qui s'annonce dans les années 1920 a un double caractère. En premier lieu elle comporte, «dans le quotidien du syndicalisme», l'adoption de pratiques syndicales qui sont celles également des internationaux. "Les deux mouvements cherchaient à conclure des ententes avec les employeurs par le truchement de la convention collective; ils utilisaient tous les deux en guise de moyens de pression sur le patronat l'atelier fermé, l'étiquette syndicale et la grève.» (249) En voie de devenir une organisation revendicative, «la centrale radicalisa ses options»: ce fut là le deuxième aspect de la mutation en cours. Il ne faut pas en exagérer la portée (ce que Rouillard ne fait certes pas); mais on ne saurait la négliger non plus. 
Ce radicalisme est surtout refus, dans la pratique, de se laisser guider par la perspective illusoire et utopique d'une "conversion des milieux industriels», qui remplacerait par un "esprit nouveau» de collaboration les rapports conflictuels entre patrons et ouvriers (249). D'où un fossé qui se creuse entre «l'idéologie formulée par les syndicats catholiques et leurs pratiques syndicales. On prêchait l'harmonie entre le capital et le travail... et pourtant on négociait des conventions collectives dans un esprit de rapports de force». D'où une analogie entre les pratiques syndicales et les réclamations de la CTCC et du CMTC qui, «issus d'un courant idéologique tout à fait différent, en étaient venus dans le quotidien du syndicalisme à se ressembler étrangement» (248-249). Il y a là, en puissance au moins, un élément important de convergence.

Pour l'auteur, le vocable "Syndicats nationaux» comprend tout ce qui n'est pas embrigadé sous la bannière de la Fédération américaine du travail (A.F.L.). C'est peut-être un peu rapide. Les Chevaliers du travail, que dirigeait Terence Powderley, formaient une composante importante du mouvement ouvrier aux États-Unis bien avant d'être perçus, rétrospectivement, comme ayant été des syndicats «nationaux, canadiens». Le syndicalisme catholique, pour sa part, avait sa source doctrinale au Vatican, avec lequel d'ailleurs ses liens politico-organisationnels autant que spirituels demeurent importants tout au long de la période étudiée. Au caractère international de ces deux courants du syndicalisme, on peut ajouter, en contre-partie, cette nuance pour ce qui est des «unions internationales» (dites «américaines»): à côté de leur assujettissement aux bureaux-chefs d'outre-frontière, avec les empiétements souvent scandaleux auxquels il donne lieu, il existe un certain phénomène de "naturalisation» (au sens d'une acquisition de citoyenneté, d'acculturation).

C'est dans un contexte international que se constitue le national. L'industrialisation capitaliste en Amérique du nord se fait à partir de la métropole britannique d'abord, pour se consolider ensuite à l'intérieur des frontières des États-Unis et du Canada, sur une échelle et à des rythmes fort inégaux. À la dépendance des colonies de l'Amérique du nord britannique, constituées en étatDominion à peine un siècle après la création des États-Unis, est surajoutée une dépendance économique à l'égard du colosse américain; tandis qu'à l'intérieur du Canada c'est le rapport d'inégalité entre communautés anglophone et francophone qui fonde une dualité historique. De tout cela, le mouvement naissant des travailleurs industriels du continent portera l'empreinte. Et de double façon. 
Car d'une part le regroupement d'ouvriers soucieux de défendre leurs intérêts s'est fait tout d'abord sur le terrain canadien ou québécois - le lieu même de leur exploitation; ce qui n'exclut point des liens externes (avec quelques syndicats britanniques dès les années 1850, avec plusieurs syndicats américains dès les années 1860). D'autre part, s'agissant de rapports avec les patrons propriétaires des moyens du travail, détenteurs du capital, il se tissera des liens de dépendance et d'antagonisme, expression de rapports de propriété, de travail et d'exploitation. Et tout cela traversera de part en part tant les rapports nationaux qu'internationaux.

Aux États-Unis, la fondation de la National Labor Union (1866) et des Chevaliers du travail (1869) affirma une volonté de «libérer le travail de l'assujettissement capitaliste», de résister aux forces coalisées du capital, d'assurer au travail «l'obtention de la juste part des richesses qu'il a créées». Dans le cas des Chevaliers, le but proclamé d'un dépassement du système de salariat s'accompagne de l'expression d'un souhait d'"harmonisation des intérêts du travail et du capital» ${ }^{1}$. La tentative faite un peu plus tard en vue de former une Canadian Labor Union, et l'implantation en ce pays d'assemblées des Chevaliers du Travail, traduisent non seulement l'exercice d'influences américaines, mais surtout la présence de rapports sociaux conflictuels, à l'oeuvre dans chacun des pays. C'est ce double aspect du phénomène syndical (et plus tard, politique, socialiste) que l'approche par le seul «national» n'arrive pas à saisir.

«Le nationalisme que le patronat invoquait... servait en fait de paravent à sa lutte contre le principe même de la négociation collective» (146).

«L'orientation plus militante de la CTCC dressa contre elle les classes dirigeantes dont une bonne partie du clergé...» (246).

Le facteur national ne saurait se situer de façon adéquate tant qu'on le traite en dimension homogène, non différenciée. C'est pourtant ce qui arrive à plusieurs reprises dans Les syndicats nationaux... De sorte que les affirmations citées plus haut font plutôt figure d'exception. Et pourtant, l'opposition structurée, imbriquée au centre même de l'ordre social, et qui dresse les ouvriers face aux employeurs, est bien présente, quoiqu'en sourdine, tout au long de l'ouvrage. Où donc est le problème?

1 CF. Philip Foner, History of the Labor Movement in the United States (1947, 1955, New York): 1, ch. 21: 433-438; ch. 25: 504-512; 2, ch. 3-4: 47-92, ch. 11: 157-170. 
D'une part, il y a le flou conceptuel: par exemple, vouloir remplacer le vocable «nationaliste» par celui de «cléricoconservateur», tout en invoquant (comme le fait l'auteur à la page $254 n$ ) la soi-disant impossibilité de donner au terme «national» un sens bien arrêté, univoque, n'est guère satisfaisant. Au pire, on peut "décréter» sa propre définition, quitte à en faire dès lors un usage conséquent. C'est de ne pas s'y résoudre qui rend certaines autant la frustration méthodologique que l'incertitude conceptuelle.

D'autre part, tout aussi malcommode est la vague sensation ressentie par le lecteur (par celui-ci, du moins), d'une absence de raccordement entre les domaines, pourtant étroitement reliés, de la culture, du socio-économique, et du politique-étatique. Exemple: l'Église catholique est prise comme un donné, qui va de soi. Ses liens avec la propriété foncière et commerciale-industrielle, et la traduction de ces liens en pouvoir dominant; la dimension culturelle-idéologique, traduite en attitudes de soumission résignée, mais génératrice - comme le sont d'ailleurs les liens de propriété et du pouvoir de classe, dominant et omniprésent - de réflexes contestataires et d'utopies: tout cela est soi absent, soit effleuré au passage. On le verra mieux dans la conclusion de l'ouvrage.

Côté idéologie, arrêtons-nous un instant sur le tableau que nous présente l'auteur de la juxtaposition des conceptions du monde propagées respectivement par les «nationaux-catholiques» et les «internationaux». C'est là une partie en quelque sorte déterminante de l'argument d'ensemble que propose Rouillard, et elle est riche en renseignements et en pistes d'analyse. Dégageons-en quelques éléments.

La distinction, le contraste fondamental, entre «catholiques» et «internationaux» se retrouverait au niveau du fait national. D'accord. Le mérite de l'auteur est d'avoir donné à ce fait l'importance qui lui revient, surtout en ce qui concerne l'émergence et la consolidation du courant syndical catholique, et l'imposition de limites à la croissance des «unions internationales». Les rapports conflictuels de ces deux courants majeurs s'en trouvent éclaircis, documentés. Ce qui eût pu nous instruire encore davantage là-dessus, aurait été l'approfondissement de deux facettes de ce pattern contradictoire: (1) le rôle d'appoint joué par le syndicalisme international («américain» ou «nord-américain») en étayant à la fois la structure fédérale canadienne et son fonctionnement en 
tant que courroie de transmission au service du grand capital canado-américain, dont l'emprise sur le pays est en rapide croissance depuis la première guerre mondiale; et (2) le dénominateur commun, présent de façon différenciée mais présent quand même dans les deux courants syndicaux, d'une orientation dominante axée sur la collaboration des classes. (Nous y reviendrons). Cette orientation rend acceptable, «respectable», un syndicalisme qui s'insère dans le système commercial. L'exemple de la négociation autour de l'étiquette syndicale en fournit un cas d'espèce frappant.

Mais attention! - le phénomène de l'organisation des travailleurs à gages, au service du capital, demeure contradictoire. La composante de la lutte sociale ne peut être éludée. Les conditions matérielles et spirituelles-culturelles des familles ouvrières sont telles (à cette époque-là et de nos jours encore) que le militantisme et la radicalisation sont inéluctables. Ce qui fait que l'explication (253) par «matérialisme», élément de l'idéologie libérale américaine, n'est guère convaincante. Qu'il s'agisse de la vision «productiviste», ou de la société dite de «consommation», soit. Mais si on la laisse là, sans évoquer l'impulsion autrement plus urgente (et valable dans une toute autre optique), qui vise l'acquisition de nourriture, de logement, de conditions de santé au travail et dans les quartiers ouvriers - et qui sont régulièrement sacrifiées aux impératifs du profit patronal - alors, on risque d'être pris au piège d'un «spiritualisme» qui camoufle le réel de la pauvreté ainsi que le vol institutionnalisé que pratique le régime des grandes corporations d'affaires.

En conclusion, Jacques Rouillard met de l'avant une interprétation d'ensemble des processus complexes qui ont marqué l'évolution du mouvement ouvrier au Québec: «Les importants conflits intersyndicaux qui ont caractérisé la période étudiée reflètent les difficultés des travailleurs à accepter certaines contraintes liées à l'industrialisation.» Suite au passage de la production artisanale à l'industrie moderne, à la disparition du lien personnel entre ouvrier et patron, les travailleurs ont recours au syndicalisme. De profondes divergences d'orientation parmi les quatre groupes étudiés font qu'ils se rattachent «à deux grandes familles idéologiques: les uns acceptant les contraintes liées à l'industrialisation»" - ce sont les internationaux et les syndicats nationaux (nonconfessionnels) - «et les autres se référant à des schémas d'action pré-industriels» - ce sont les Chevaliers du travail et les syndicats catholiques (307).

Les premiers «ne remettent pas fondamentalement en cause ni l'industrialisation comme telle ni le système capitaliste qui l'a générée». De cette conception découle le «business unionism», syndica- 
lisme d'affaires, auquel se rattache le nom de Gompers. Ce sont les relations du travail définies dans les paramètres de la négociation collective d'un "contrat de travail négocié librement avec chaque employeur». Il y a "perpétuel conflit» entre deux groupes «à la fois concurrents et interdépendants», dont les rapports de force peuvent varier, tout en demeurant à l'intérieur d'une structure de collaboration marchande, capitaliste.

Les seconds conservent la nostalgie des rapports, supposément idylliques, de l'époque préindustrielle. Ils comptent pouvoir en arriver à l'harmonie du capital et du travail, soit par la diffusion de projets coopératifs, soit par la diffusion des principes chrétiens de charité sociale, aboutissant à «une transformation morale des individus» (311).

Dans les deux cas, c'est la promotion d'une collaboration de classes antagoniques qui se dégage comme thème dominant: que ce soit le syndicalisme d'affaires bien pragmatique, ou l'utopie idéaliste et idéalisante. C'est ce qui semble se dégager des données présentées par l'auteur, sans qu'il le formule de manière explicite. Son dernier mot étant:

Les sociétés industrialisées de type libéral laissent peu d'autres choix aux travailleurs que de concevoir leurs relations avec l'employeur sous le signe d'un rapport de forces. Le modèle corporatiste méconnaissait cette règle; il relevait d'une idéologie complètement en désaccord avec une société industrialisée. (312)

Le point faible, à mon sens, consiste à vouloir limiter l'éventail des options à un passé révolu ou bien à un présent immuable. Comme si personne, où que ce soit, n'ait jamais songé à un avenir qui soit un dépassement du capitalisme existant. Et pourtant, les impulsions vers une radicalisation, évoquées par Rouillard à plus d'une reprise, n'exclurent guère la possibilité de ce qui, effectivement, est arrivé au cours du dernier demi-siècle; l'émergence, dans la conscience des catholiques, de l'idée d'une éventuelle restructuration en profondeur de l'ordre social, où les «principes chrétiens» seraient autre chose que leurre ou utopie. De Rerum novarum à Pacem in terris il se trace une trajectoire, non pas univoque ni unilinéaire, mais qui recèle un potentiel de choix qui transcende les paramètres figés d'un monde capitaliste fondé sur l'exploitation ad vitam aeternam.

La problématique que nous propose Rouillard est celle d'une adaptation de la classe ouvrière québécoise francophone aux institutions capitalistes nord-américaines du début du 20e siècle. Qu'il y ait là un aspect important du vécu historique, nul doute possible. 
Mais ce n'est pas tout: et le fait de sembler accepter comme une donnée immuable le rapport capital-travail, rapport d'exploitation, et donc d'antagonisme et de lutte, a pour effet d'amputer du récit une dimension de taille. Car le fait est que la syndicalisation des ouvriers constitue non seulement une réplique défensive aux empiétements des patrons, mais aussi (en puissance d'abord, en réalité ensuite), une remise en question du salariat. C'est ce côté «subversif» que l'apologétique dominante est portée à dévaloriser ou à taire. (On songe aux jugements un tantinet hautains de Fernand Dumont ou de Fernand Harvey, alléguant soit la nonexistence soit la non-signification de velléités socialistes ou d'action politique ouvrière avant les années 1960) ${ }^{2}$.

Dans la crise que traverse actuellement la Pologne, on constate la profondeur des dimensions enchevêtrées du national, du catholique et du social. Un mouvement syndical qui confronte un pouvoir de socialisme d'État dominé de l'extérieur: certes, ce contexte spatio-temporel est fort différent et bien éloigné de celui du Québec des années 1900-1930; et pourtant, il reste un brin de dénominateur commun. On peut le déceler dans l'entrecroisement de facteurs à trois niveaux: ceux de l'ordre socio-économique (et son expression étatique), de la communauté nationalitaire, et de l'idéologie. À condition de tenir bien présente à l'esprit toute l'ampleur de la différence historique, il est peut-être admissible de poursuivre, brièvement, cette analogie.

Au plan de l'idéologie, c'est la dimension catholique qui dans les deux cas joue un rôle primordial, face à la pression envahissante d'une idéologie adverse: protestante dans un cas, marxisteléniniste dans l'autre, chacune émanant de la société voisine, jugée «matérialiste», accapareuse, menaçante. Le «national» s'y retrouve déjà en tant que facteur d'opposition majeure: Polonais versus Russes, "Canadiens français» versus «Anglais» (AngloCanadiens ou Américains) - c'est à chaque fois un petit peuple qui confronte un voisin plus puissant, sur une toile de fond historique d'une opposition de longue durée.

S'arrêter là, ce serait commode. Mais reste toujours la question de l'ordre social, de la structure économique et étatique, des classes sociales. Les aspects idéologiques et nationalitaires s'y rattachent, voire s'y enracinent. L'Église, comme le Parti, traduit des intérêts tant socio-économiques que politiques, intérêts cristallisés

\footnotetext{
2 F. Dumont, Idéologies du Canada français 1900-1929, 7 n; F. Harvey, Le mouvement ouvrier au Québec (1980), 47.
} 
dans le cadre étatique, tout en le dépassant, et de loin. À ce niveau la divergence des cas s'impose de plus en plus: entre les mondes ancien (du début de ce siècle) et actuel, que reste-t-il de commun? Et quel est le caractère de l'écart qui les sépare? Le phénomène de l'industrialisation, de l'émergence et de l'affirmation d'une classe ouvrière, et de son expression syndicale et politique, est présent, quoique la spécificité historique, dans les deux cas, soit fort dissemblable. De sorte qu'on est amené à insister sur celle-ci: au point où disparaitrait toute ressemblance? Pas si certain... 